\title{
Evaluation of Antimicrobial Activity of Organic Acids against Campylobacter jejuni in Broilers
}

\author{
Faiza Ghazanfar ${ }^{1}$, Masood Rabbani ${ }^{1 *}$, Aamir Ghafoor $^{2}$ and \\ Muhammad Hassan Mushtaq ${ }^{3}$ \\ ${ }^{1}$ Institute of Microbiology, University of Veterinary and Animal Sciences, Lahore \\ ${ }^{2}$ University Diagnostic Laboratory, University of Veterinary and Animal Sciences, \\ Lahore \\ ${ }^{3}$ Department of Epidemiology, University of Veterinary and Animal Sciences, Lahore
}

\begin{abstract}
A B S T RA C T
Campylobacteriosis is a collective term used for the infection caused by the members of Campylobacter species. The causative agent is Campylobacter that asymptomatically colonizes broilers during development and contaminates it during slaughter. Outbreaks mostly start from the ingestion of contaminated poultry products or infected water. Reducing colonization of Campylobacter jejuni in the gut can be useful in decreasing the contamination of the poultry. Different organic acids display potential as a substitute of antibiotics. These not only improve poultry performance by modifying the $\mathrm{pH}$ of the gastro-intestinal tract of bird, but also change the composition of its microbiome and ultimately protecting the chicken from $\mathrm{pH}$-sensitive pathogens. The purpose of this study was to define the bactericidal action of organic acids on Campylobacter jejuni, individually and in combination. Total 120 broiler chickens were randomly distributed in ten groups. The groups included negative and positive control, pure organic acid group and commercial organic acid formulation group. Excluding negative control group, all other groups were orally challenged with $0.1 \mathrm{ml}$ of the 6-Log $10 \mathrm{CFU} / \mathrm{ml}$ of the Campylobacter jejuni culture in normal saline via oral route. Cloacal samples were collected for Campylobacter count, body weight (BW) and feed conversion ratio (FCR), which were determined weekly and cumulatively for 35 days. The birds of a specific treatment group were given organic acid on daily basis for 6-8 $\mathrm{h}$. Excluding the negative control group, all groups were tested with fresh culture of Campylobacter jejuni on 14, 21, 28 and 35 day of age. Bacterial count was performed at $6,8,13,15,20,22,27,29,34$ and 36 day of age. The results suggest synergistic actions of a mixture of organic acids are effective for decreasing Campylobacter jejuni colonization in vivo. Moreover, our study also suggests that there is no direct impact of organic acids on weight gain and FCR of the birds statistically.
\end{abstract}

Article Information

Received 09 August 2021

Revised 07 October 2021

Accepted 19 October 2021

Available online 04 February 2022

(early access)

\section{Authors' Contribution}

FG, MR, AG and HM conceived and designed study. FG executed the experiments. FG and MR analyzed the data. FG and MR prepared the manuscript. The manuscript is critically revised by all authors and approved this final version of manuscript.

Key words

Campylobacter jejuni, Organic acid, Broiler chicken, Feed water, Bacteria, Food safety, Poultry

\section{INTRODUCTION}

C ampylobacter jejuni is recognized to be the prominent cause of human intestinal ailments globally (Gharib et al., 2012). Poultry and poultry products are identified to be the main reservoir of this bacterium (Jørgensen et al., 2002). Campylobacter infections may be fatal in children, aged and immuno-compromised patients (Beier et al., 2020). An infective dosage of only around 500-800 of $C$. jejuni are required for infection (Kothary and Babu, 2001). Using replacement ways and means to prevent colonization of these bacteria in the intestinal tract of flocks may help regulate the spread of these bacteria from food to human (Rosenquist et al., 2006).

Certain organic acids have long been used as food

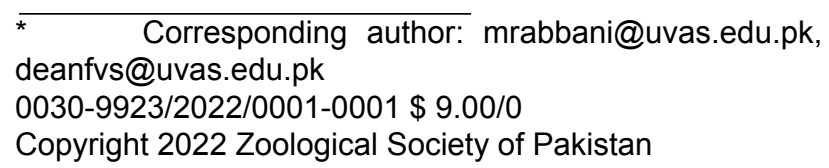

condiments and for extending the shelf life of perishable food constituents. Fatty acids have been described to possess antimicrobial activities against a wide range of microorganisms (Hermans et al., 2011). The mechanism of organic acid inhibition is presumed to be principally dependent on $\mathrm{pH}$ (Nannapaneni et al., 2009) or the undissociated arrangement of the organic acids (Fernández and Pisón, 1996), which are thought to penetrate the lipid membrane. Nevertheless, the precise mechanism by which organic acids constrain bacteria is not known (Beier et al., 2020). It has furthermore been stated that using organic acids in drinking water lessens $C$. jejuni population in crop and carcasses (Byrd et al., 2001).

The purpose of this study was to examine the bactericidal effects of different organic acids, either single on in a mixture, in reducing cecal colonization and excretion of $C$. jejuni if given through drinking water. Body weight gain and feed conversion ratio were also monitored. 


\section{MATERIALS AND METHODS}

\section{Animal housing}

A total of 120 broiler chicks were acquired from a commercial hatchery on the day of hatch and raised for 35 days in the experimental chamber of animal shelter, Institute of Microbiology, UVAS, Lahore. The primary weight of the hatchlings was $40.0-41.35 \mathrm{~g}$. The cloacal swabs in normal saline were taken and grown on CCDA under microaerophillic conditions to confirm the chicks are Campylobacter free. The chicks were kept on floor pens with wood shavings. The composition and nutrient value of the basal diet is described in Table I. Feed and water were accessible ad libitum for the 35-day trial.

Table I. The composition and nutrient values of basal $\operatorname{diet}(\%)$.

\begin{tabular}{lll}
\hline Ingredients & Starter (1-17 Days) & Grower (18-35 Days) \\
\hline C.P & 22.7 & 21.2 \\
Fat & 4.1 & 4.6 \\
Ash & 3.6 & 4.0 \\
M.E & 2900 & 2980 \\
Ca & 0.9 & 1.0 \\
P & 0.52 & 0.46 \\
D. Lysin & 1.2 & 1.16 \\
D. Methionine & 0.5 & 0.464 \\
D. Threonine & 0.79 & 0.76 \\
\hline
\end{tabular}

\section{Microbial strain}

C. jejuni strain ATCC 33291, used for inoculation of the birds, was kept frozen at $-80^{\circ} \mathrm{C}$ in $80 \%$ glycerol solution. The innoculum was prepared for the challenge trial by culturing it on Charcoal-CephoperazoneDeoxycholate-Agar (CCDA) and incubating for $48 \mathrm{~h}$ at $42^{\circ} \mathrm{C}$ under microaerophillic conditions $\left(5 \% \mathrm{O}_{2}, 10 \% \mathrm{CO}_{2}\right.$ and $85 \% \mathrm{~N}_{2}$ ). The bacteria were harvested and diluted in normal saline solution to the precise viable concentration of 6-Log $10 \mathrm{CFU} / \mathrm{ml}$ according to the technique explained by (Lamb-Rosteskiet al., 2008). Inoculum concentration was assessed by $0.1 \mathrm{MacFarland}$ tubes. The inoculum was kept on ice for less than $1 \mathrm{~h}$ before oral administration of chicks. Except the negative control group, the rest of the birds received $1 \mathrm{ml}$ dose of the inoculum in normal saline at day 7 of the trial (Table II).

\section{Organic acids as an alternate therapy}

Four pure organic acids (Formic Acid, Propionic Acid, Acetic Acid and Lactic Acid) and four commercially available preparations i.e. Multiacid (EWABO, The
Hygiene Company, Wietmarschen, Germany) composed of formic acid, acetic acid, propionic acid and lactic acid, SELKO-pH (Selko Feed Additives, Tilburg, Netherlands) made up of ammonium formate, formic acid and acetic acid, Acid Punch (Herbavita Feed Supplements, ZS Biotech, Pakistan) consisted of formic acid, propionic acid, acetic acid and lactic acid and Lipto-Safe L (Forward Solutions, Pakistan) contained formic acid, propionic acid, citric acid and lactic acid, were used in treatment groups to check the reduction in microbial count. The $\mathrm{pH}$ of all acids and formulations were adjusted at level 4 .

\section{Experimental design}

The chicks were randomly distributed to eight treatment groups containing 10 birds in respective groups. The birds of the particular group were given respective organic acid on daily basis in drinking water. $C$. jejuni lives as a commensal in chicken gut. Therefore, the microbial load of this pathogen was estimated by taking cloacal swab in normal saline and growing on CCDA under microaerophillic conditions, a day before administering the fresh culture of this bacterium. The initial count was noted down. Except the negative control group, all treatment groups were tested with fresh culture of $C$. jejuni (0.1 MacFarland) on 14, 21, 28 and 35 day of age. The bacterium was allowed $24 \mathrm{~h}$ to colonize the gut of the bird. After $24 \mathrm{~h}$, the microbial load was again calculated by taking the cloacal swab in normal saline and growing the sample on CCDA under microaerophillic conditions, a day after administering the innoculum. The reading was again noted down and compared with the initial count. The study plan is presented in Table II. Chickens of all groups were vaccinated against New Castle Disease (NDV) vaccine according to the routine vaccination program of the broilers which contains administration of live virus "LaSota" vaccine via eye drop route at day 5 followed by booster dose at day 15 .

\section{Weight gain analysis on weekly basis}

The results of different organic acids, affecting the body weight gain (BW) of the birds of all groups, were obtained on weekly basis that is on day 1, 7, 14, 21, 28 and 35 . Whereas, feed intake and water consumption were checked on daily basis. For 35-day trial, $2 \mathrm{~kg}$ feed was given to all groups for $24 \mathrm{~h}$ every day. Feed conversion ratio (FCR) was determined by the intake of the feed vs weight gain of the bird.

\section{Microbial count}

Microbial load for $C$. jejuni was evaluated from each bird by collecting the cloacal swab in normal saline at 6,8 , $13,15,20,22,27,29,34$ and 36 day of age. The samples 
were diluted serially ten-fold and counted on CCDA under microaerophillic conditions. Bacterial colonies were counted and CFU/gram was converted into $\log _{10}$ values. The mean \pm standard deviation (S.D) of $\log _{10}$ values were calculated and compared among groups. Log reduction of plate count was calculated by subtracting log values of day post infection (DPI) from day before infection (DBI).

\section{Statistical analysis}

The data was transferred to the spreadsheet using MS Excel 2016 and the results were evaluated through
Statistical Package for the Social Sciences (SPSS) version 16.0. Enumeration data was presented as Mean \pm S.D $\log _{10}$ $\mathrm{CFU} / \mathrm{mL}$ and compared by one-way ANOVA followed by Tukey's multiple comparison test at $p<0.05$ level of significance by SPSS.

\section{RESULTS AND DISCUSSION}

The control strategies on-farm, for the decrease of Campylobacter, have been comprehensively studied as a significance of well-established association of

Table II. Treatment group description (organic acid/distilled water) at pH 4.

\begin{tabular}{|c|c|c|}
\hline \multicolumn{2}{|c|}{ Groups } & Treatment \\
\hline \multicolumn{3}{|c|}{ Control groups } \\
\hline A & Negative control & No treatment \\
\hline B & C. jejuni & C. jejuni treatment we \\
\hline \multicolumn{3}{|c|}{ Pure organic acid model } \\
\hline $\mathrm{C}$ & Formic acid $(0.1 \mathrm{~mL} / 1 \mathrm{~L})$ & Daily $+C$. jejuni treatment weekly \\
\hline $\mathrm{D}$ & Acetic acid $(0.1 \mathrm{~mL} / 100 \mathrm{~mL})$ & Daily $+C$. jejuni treatment weekly \\
\hline $\mathrm{E}$ & Propionic acid $(0.1 \mathrm{~mL} / 100 \mathrm{~mL})$ & Daily + C. jejuni treatment weekly \\
\hline $\mathrm{F}$ & Lactic acid $(0.1 \mathrm{~mL} / 130 \mathrm{~mL})$ & Daily + C. jejuni treatment weekly \\
\hline \multicolumn{3}{|c|}{ Commercial organic acid model } \\
\hline G & Acid punch $(0.25 \mathrm{~mL} / 1000 \mathrm{~mL})$ & Daily+C. jejuni treatment weekly \\
\hline $\mathrm{H}$ & Lipto-Safe L $(0.1 \mathrm{~mL} / 250 \mathrm{~mL})$ & Daily $+C$. jejuni treatment weekly \\
\hline I & SELKO-pH $(0.1 \mathrm{~mL} / 180 \mathrm{~mL})$ & Daily $+C$. jejuni treatment weekly \\
\hline $\mathrm{J}$ & Multiacid $(0.1 \mathrm{~mL} / 200 \mathrm{~mL})$ & Daily $+C$. jejuni treatment weekly \\
\hline $\mathrm{K}$ & Antibiotic (Ciprofloxacin) & Antibiotic formulation $+C$. jejuni treatment weekly \\
\hline
\end{tabular}

Table III. Antibacterial activity of organic acids against Campylobacter jejuni.

\begin{tabular}{|c|c|c|c|c|c|c|c|c|c|c|c|}
\hline \multirow[t]{2}{*}{ Groups } & \multirow{2}{*}{$\begin{array}{l}6(\mathrm{BC}) \\
\text { Count }\end{array}$} & \multicolumn{2}{|c|}{ Day 7} & \multicolumn{2}{|c|}{ Day 14} & \multicolumn{2}{|l|}{ Day 21} & \multicolumn{2}{|l|}{ Day 28} & \multicolumn{2}{|l|}{ Day 35} \\
\hline & & Count & L.D & Count & L.D & Count & L.D & Count & L.D & Count & L.D \\
\hline A & 3.41 & 3.44 & - & 3.33 & - & 3.31 & - & 3.77 & - & 3.66 & - \\
\hline B & 3.37 & 6.55 & - & 6.45 & - & 6.27 & - & 6.22 & - & 6.15 & - \\
\hline $\mathrm{C}$ & 3.05 & 4.16 & 3.19 & 3.88 & 2.17 & 3.23 & 2.82 & 3.61 & 2.44 & 3.77 & 2.28 \\
\hline $\mathrm{D}$ & 3.18 & 4.32 & 2.23 & 3.32 & 2.9 & 3.19 & 2.31 & 3.17 & 3.08 & 3.05 & 2.6 \\
\hline $\mathrm{E}$ & 3.15 & 5.07 & 1.48 & 5.01 & 1.54 & 4.77 & 1.45 & 4.92 & 1.63 & 4.61 & 1.94 \\
\hline $\mathrm{F}$ & 3.35 & 4.72 & 1.83 & 4.61 & 1.94 & 4.38 & 1.84 & 4.05 & 1.45 & 4.01 & 2.63 \\
\hline G & 3.33 & 3.93 & 2.12 & 3.77 & 2.28 & 3.75 & 2.3 & 3.61 & 2.44 & 3.23 & 2.82 \\
\hline $\mathrm{H}$ & 3.27 & 4.01 & 2.23 & 4.06 & 2.16 & 3.82 & 2.45 & 3.75 & 2.4 & 3.61 & 2.54 \\
\hline I & 3.39 & 4.38 & 1.84 & 4.05 & 2.17 & 4.12 & 2.1 & 4.24 & 2.83 & 4.16 & 2.26 \\
\hline $\mathrm{J}$ & 3.33 & 4.61 & 1.94 & 4.77 & 1.45 & 3.93 & 2.34 & 3.82 & 2.45 & 3.77 & 2.38 \\
\hline K & 3.29 & 4.88 & 1.67 & 4.86 & 1.54 & 4.06 & 2.16 & 3.82 & 2.45 & 3.75 & 2.4 \\
\hline
\end{tabular}

Count, Mean \pm SEM $\log ^{10} ;$ LD, $\log ^{10}$ reduction of C. jejuni. 
Campylobacter and poultry meat (Santini et al., 2010; Gharib et al., 2012; Neal-McKinney and Konkel, 2012; Nishiyama et al., 2014) Studies designate that adding organic acid to the drinking water aids in the reduction of pathogens in the water and the crop/ proventriculus, to control gut micro-flora, to intensify the digestion of feed and to increase growth performance (Byrd et al., 2001; Açıkgöz et al., 2011; Hamed and Hassan, 2013). However, the exact mechanism(s) by which organic acids prevent bacteria are not known (Kim et al., 2019).

In this project, we compared the bactericidal effect of organic acids on load of $C$. jejuni at $\mathrm{pH}$ level 4. The outcomes of this experiment demonstrate that the acidification of the drinking water successfully reduced the number of $C$. jejuni in the guts of the experimental birds. The antibacterial activity of organic acids against $C$. jejuni at different weeks of 35 days trial is presented in the Table III. The effect of organic acids in reduction of $C$. jejuni is presented in Figure 1.

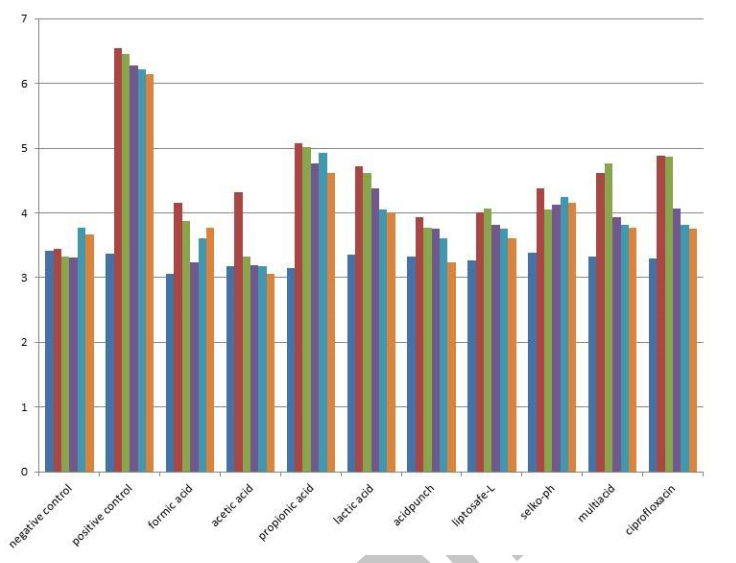

Fig. 1. Growth of C. jejuni on selective media (CCDA).

The main objective of our study plan was to evaluate the effect of organic acids on $C$. jejuni either already present in the chicken gut or administered orally. The experimental trial was designed to choose for an effective bactericidal method; whether it's the use of an individual organic acid as a better controlling measure or should it be a mixture of organic acids that obstructs the colonization of $C$. jejuni in the chicken gut.

Although all treatment groups show a decrease of $C$. jejuni, the treatment groups involving the combination of organic acids gave better results. Group G (Acid Punch) lower the load in the steadiest manner, followed by group $\mathrm{H}$ (LiptoSafe-L). No significant increase in the body weight of the birds was noted during the first 14 days of the trial. After the 2 weeks, positive control versus negative control showed differences in body weight gain.
Weekly average weight gains of birds throughout the 35 days trial is presented in Table IV, while weekly FCR of experimental groups is presented in Table $\mathrm{V}$.

Table IV. Weekly average weight gain of birds in grams.

\begin{tabular}{lllllll}
\hline Groups & Day 0 & Day 7 & Day 14 & Day 21 & Day 28 & Day 35 \\
\hline A & 41.00 & 263.31 & 407.46 & 958.47 & 1421.37 & 1912.28 \\
B & 41.25 & 273.12 & 429.34 & 977.29 & 1431.27 & 1960.38 \\
C & 40.35 & 269.65 & 434.65 & 968.48 & 1464.17 & 1954.18 \\
D & 41.29 & 278.76 & 469.13 & 1023.48 & 1502.36 & 1983.37 \\
E & 40.00 & 259.46 & 434.64 & 996.39 & 1554.18 & 1918.19 \\
F & 41.30 & 252.43 & 465.21 & 1002.38 & 1535.26 & 1949.33 \\
G & 41.14 & 288.87 & 456.19 & 1050.36 & 1575.32 & 2017.28 \\
H & 41.35 & 265.69 & 446.45 & 960.10 & 1521.25 & 2048.19 \\
I & 40.16 & 282.43 & 432.32 & 1022.28 & 1536.18 & 1922.26 \\
J & 41.05 & 278.56 & 406.47 & 1087.36 & 1518.16 & 1951.66 \\
K & 40.26 & 269.47 & 442.34 & 1058.47 & 1671.14 & 2069.34 \\
\hline
\end{tabular}

Table V. Weekly FCR of experimental groups.

\begin{tabular}{llllll}
\hline Groups & Day 7 & Day 14 & Day 21 & Day 28 & Day 35 \\
\hline A & 0.94 & 1.13 & 1.33 & 1.42 & 1.69 \\
B & 0.97 & 1.16 & 1.33 & 1.46 & 1.63 \\
C & 0.97 & 1.14 & 1.39 & 1.43 & 1.59 \\
D & 0.93 & 1.06 & 1.31 & 1.42 & 1.46 \\
E & 0.96 & 1.10 & 1.36 & 1.44 & 1.55 \\
F & 0.90 & 1.10 & 1.34 & 1.41 & 1.46 \\
G & 0.91 & 1.13 & 1.32 & 1.42 & 1.42 \\
H & 0.95 & 1.12 & 1.31 & 1.42 & 1.46 \\
I & 0.93 & 1.10 & 1.34 & 1.46 & 1.60 \\
J & 0.95 & 1.06 & 1.33 & 1.43 & 1.55 \\
K & 0.96 & 1.13 & 1.36 & 1.42 & 1.65 \\
\hline
\end{tabular}

After the conclusion of 6 weeks, the weight gains varied considerably $(\mathrm{P}<0.05)$ among treatments. The maximum weight gain (2069.3 g) was noted down for group $\mathrm{K}$ which includes antibiotic ciprofloxacin. Organic acid treatment groups, composed of individual acids and mixtures of these individual acids, are found to execute anti-microbial activities comparable to those of antibiotics (Wang et al., 2009). The $\mathrm{K}$ group was for comparison sake with the outcomes of organic acid treatment groups. The results of group $\mathrm{K}$ was followed by treatment group $\mathrm{H}$ containing commercial product LiptoSafe-L (2048.1 g) and group G comprising Acid Punch (2017.2 g), showing synergistic effect of the organic acids is more favorable 
than the use of individual acids. No significant difference in terms of body weight was observed in the negative control group A (1912.2 g) and positive control group B (1960.3 g).

Chicks of the group $\mathrm{G}$ (Acid Punch) displayed a significant improvement $(\mathrm{P}<0.05)$ in terms of FCR (1.42) as compared with the chicks of group H (LiptoSafe-L), group F (Lactic acid) and group D (Acetic acid) with the same FCR (1.46). Why group H (LiptoSafe-L) did not gave better results in terms of FCR may be because $C$. jejuni cannot utilize the citric acid cycle to yield energy but it can use citric acid cycle intermediates, acetic acid and lactic acid to produce energy. The progress in the FCR in group $\mathrm{G}$ (Acid Punch) could be possibly because of the improved utilization of the nutrients causing increased body weight gain.

There was a statistically significant difference between the treatment groups at the $p<0.05$ level of significance as demonstrated by one-way ANOVA $(\mathrm{F}(10,44)=29.8$, $p=0.00)$ Details in supplementary data S1 A Tukey's post hoc test showed that there is significant difference in the group means of negative control group (Group 1) to positive control group (Group 2; sig $=0.00$ ), propionic acid group (Group 5; sig $=0.00)$, lactic acid group (Group $6 ;$ sig $=0.10)$ and antibiotic group (Group 11; $\operatorname{sig}=0.027$ ) Positive control group (Group 2) to all groups ( $\mathrm{sig}=0.00)$. Formic acid group (Group3) to positive control group (Group 2; sig = 0.00) and lactic acid group (Group 5; sig $=0.00)$. Acetic acid group (Group 4) to positive control group (Group 2; sig =0.00), propionic acid group (Group 5 ; sig $=0.00)$, lactic acid group (Group 6; sig $=0.03$ ), Selko-pH group (Group 9; sig = 0.025), MultiAcid group (Group 10; sig $=0.028$ ) and antibiotic group (Group 11; $\operatorname{sig}=0.008)$. Propionic acid group (Group 5) to negative control group (Group 1; sig =0.00), positive control group (Group 2; sig = 0.00), formic acid group (Group 3; sig = 0.00 ), acetic acid group (Group 4; sig $=0.00$ ), Acidpunch group (Group 7; sig $=0.00$ ) and Liptosafe group (Group 8 ; sig $=0.001)$. Lactic acid group (Group 6) to negative control group (Group 1; sig $=0.010)$, positive control group (Group 2; sig = 0.00) and acetic acid group (Group 4 ; sig $=0.003)$. Acidpunch group (Group 7) to positive control group (Group 2; sig $=0.00$ ) and propionic acid group (Group 5; sig =0.00). Lipto-safe group (Group 8) to positive control group (Group 2; sig $=0.00$ ) and propionic acid group (Group 5; sig = 0.001). Selko-pH group (Group $9)$ to positive control group (Group 2; sig $=0.00$ ) and acetic acid group (Group 4; sig =0.025). MultiAcid group (Group 10) to positive control group (Group 2; sig = 0.00) and acetic acid group (Group 4; sig $=0.028$ ). Antibiotic group (Group 11) to negative control group (Group1; sig $=0.027)$, positive control group (Group $2 ;$ sig $=0.00)$ and acetic acid group (Group 4; sig $=0.008$ ). The statistical results show that there is no significant effect of organic acids on the WG of birds or FCR.

As the bacterial colonization is reduced, it gives a positive outcome on the health of the bird specified by good health, notable weight gain and acceptable FCR. These reductions, although appeared to be very small but important, can have serious impact on poultry industry.

\section{CONCLUSIONS AND RECOMMENDATIONS}

In conclusion, this study displayed that synergistic actions of the combined organic acid supplementation (Acid Punch) presented higher decline rates of Campylobacter spp. than the single organic acids. It is not only effective in dropping the microbial count in an in vivo trial experiment, but also retains the general wellbeing by inhibiting the development of possible food borne pathogens.

\section{ACKNOWLEDGEMENTS}

Authors acknowledge Higher Education Commission (HEC) funded project\# 4333/NRPU/R\&D/HEC/14/278 entitled "Evaluation of anti- Campylobacter activity of indigenous probiotic Lactobacilli alone and in combination with different organic acids in poultry" for supporting this study.

\section{Statement of conflict of interest}

The authors have declared no conflict of interest.

\section{REFERENCES}

Açıkgöz, Z., Bayraktar, H. and Altan, Ö., 2011. Effects of formic acid administration in the drinking water on performance, intestinal microflora and carcass contamination in male broilers under high ambient temperature. Asian Aust. J. Anim. Sci., 24: 96-102. https://doi.org/10.5713/ajas.2011.10195

Beier, R.C., Byrd, J.A., Andrews, K., Caldwell, D., Crippen, T.L., Anderson, R.C. and Nisbet, D.J., 2020. Inhibition and interactions of Campylobacter jejuni from broiler chicken houses with organic acids. Microorganisms, 7: 223. https://doi. org/10.3390/microorganisms 7080223

Byrd, J.A., Hargis, B.M., Caldwell, D.J., Bailey, R.H., Herron, K.L., McReynolds, J.L., Brewer, R.L., Anderson, R.C., Bischoff, K.M., Callaway, T.R. and Kubena, L.F., 2001. Effect of lactic acid administration in the drinking water during preslaughter feed withdrawal on Salmonella and Campylobacter contamination of broilers. Poult. 
Sci. J., 80: 278-283. https://doi.org/10.1093/ $\mathrm{ps} / 80.3 .278$

Fernández, H. and Pisón, V., 1996. Isolation of thermotolerant species of Campylobacter from commercial chicken livers. Int. J. Fd. Microbiol., 29: $75-80$.

Gharib, N.K., Rahimi, S. and Khaki, P., 2012. Comparison of the effects of probiotic, organic acid and medicinal plant on Campylobacter jejuni challenged broiler chickens. J. Agric. Sci. Technol., 14 (Supp):

Hamed, D.M. and Hassan, A.M.A., 2013. Acids supplementation to drinking water and their effects on Japanese quails experimentally challenged with Salmonella enteritidis. Res. Zool., 3: 15-22.

Hermans, D., Deun, K.V., Messens, W., Martel, A., Immerseel, F.V., Haesebrouck, F., Rasschaert, G., Heyndrickx, M. and Pasmans, F., 2011. Campylobacter control in poultry by current intervention measures ineffective: Urgent need for intensified fundamental research. Vet. Microbiol., 152: 219-228. https://doi.org/10.1016/j. vetmic.2011.03.010

Jørgensen, F., Bailey, R., Williams, S., Henderson, P., Wareing, D.R.A., Bolton F.J., Frost J.A., Ward, L. and Humphrey, T.J., 2002. Prevalence and numbers of Salmonella and Campylobacter spp. on raw, whole chickens in relation to sampling methods. Int. J. Fd. Microbiol., 76: 151-164. https:// doi.org/10.1016/S0168-1605(02)00027-2

Kim, S., Jang, M.J., Kim, S.Y., Yang, Y., Pavlidis, H.O. and Ricke, S.C., 2019. Potential for prebiotics as feed additives to limit foodborne Campylobacter establishment in the poultry gastrointestinal tract. Front. Microbiol., 10: 91. https://doi.org/10.3389/ fmicb.2019.00091

Kothary, M.H. and Babu, U.S., 2001. Infective dose of foodborne pathogens in volunteers: A review. J. Fd. Safe., 21: 49-68. https://doi. org/10.1111/j.1745-4565.2001.tb00307.x

Lamb-Rosteski, J.M., Kalischuk, L.D., Inglis, G.D. and Buret, A.G., 2008. Epidermal growth factor inhibits Campylobacter jejuni-induced claudin-4 disruption, loss of epithelial barrier function, and Escherichia coli translocation. Infect. Immun., 76:
3390. https://doi.org/10.1128/IAI.01698-07

Nannapaneni, R., Chalova, V.I., Crandall, P.G., Ricke, S.C., Johnson, M.G. and O'Bryan, C.A., 2009. Campylobacter and Arcobacter species sensitivity to commercial orange oil fractions. Int. J. Fd. Microbiol., 129: 43- 49. https://doi. org/10.1016/j.ijfoodmicro.2008.11.008

Neal-McKinney, J. and Konkel, M., 2012. The Campylobacter jejuni $\mathrm{CiaC}$ virulence protein is secreted from the flagellum and delivered to the cytosol of host cells. Front. Cell. Infect. Microbiol., 2: 31. https://doi.org/10.3389/fcimb.2012.00031

Nishiyama, K., Seto, Y., Yoshioka, K., Kakuda, T., Takai, S., Yamamoto, Y. and Mukai, T., 2014. Lactobacillus gasseri SBT2055 reduces infection by and colonization of Campylobacter jejuni. PLoS One, 9: e108827. https://doi.org/10.1371/journal. pone. 0108827

Rosenquist, H., Sommer, H.M., Nielsen, N.L. and Christensen, B.B., 2006. The effect of slaughter operations on the contamination of chicken carcasses with thermotolerant Campylobacter. Int. J. Fd. Microbiol., 108: 226-232. https://doi. org/10.1016/j.ijfoodmicro.2005.12.007

Saint-Cyr, M.J., Guyard-Nicodème, M., Messaoudi, S., Chemaly, M., Jean-Michel, C., Dousset, X. and Haddad, N., 2016. Recent advances in screening of anti-Campylobacter activity in probiotics for use in poultry. Recent advances in screening of anti-campylobacter activity in probiotics for use in poultry. Front. Microbiol., 7: 553. https://doi.org/10.3389/fmicb.2016.00553

Santini, C., Baffoni, L., Gaggia, F., Granata, M., Gasbarri, R., Gioia, D.D. and Biavati, B., 2010. Characterization of probiotic strains: an application as feed additives in poultry against Campylobacter jejuni. Int. J. Fd. Microbiol., 141: S98-S108. https:// doi.org/10.1016/j.ijfoodmicro.2010.03.039

Wang, Z., Li, H., Xu, H., Xi-Ling, Y., Xiao-Qin, C., WenJun, H., Yun-Yi, Z. and Dao-Feng, C., 2009. Beneficial effect of Bupleurum polysaccharides on autoimmune disease induced by Campylobacter jejuni in BALB/c mice. J. Ethnopharmacol., 124: 481-487. 